\title{
Natureza, classificação e intervenções de enfermagem para pacientes com mediastinite
}

Nature, classification and nursing interventios for patients with mediastinitis

Naturlaleza, clasificación y intervenciones de enfermería para pacientes con mediastinitis

\author{
Amélia Rodrigues de Almeida', Maria Vilani Cavalcante Guedes' \\ 'Universidade Estadual do Ceará. Fortaleza, CE
}

Submissão: 13/03/2008

Aprovação: 3 1/07/2008

\section{RESUMO}

Trata-se de estudo Quantitativo, descritivo e retrospectivo Que objetivou analisar a natureza e classificação das intervenções de enfermagem para pacientes acometidos por mediastinite no pós-operatório de cirurgia cardíaca. Os resultados obtidos através de prontuários mostraram uma incidência de 3\% de mediastinite. A população do estudo era na maioria do sexo masculino, sendo todos diabéticos. Os problemas de enfermagem foram categorizados segundo Horta e as intervenções classificadas conforme a NIC. Dentre os problemas levantados, $76,5 \%$ foram de necessidade psicobiológica e 23,5\% psicossocial. Implementou-se 53 intervenções de enfermagem: $84,9 \%$ de natureza fisiológica, 11,3\% comportamental e 3,8\% familiar. A relação entre problemas identificados e intervenções desenvolvidas, foi adeQuada principalmente nas necessidades psicobiológicas, sendo fręüente as intervenções educativas, principalmente nas necessidades psicossociais.

Descritores: Enfermagem; Classificação; Cuidados de enfermagem; Mediastinite.

\section{ABSTRACT}

This is a descriptive and retrospective study that aimed at analyzing the nature and classification of nursing interventions for patients with mediastinitis following cardiac surgery. The results obtained from the surgical wards show an incidence of mediastinitis of $3 \%$. The subjects studies were primarily male, all of whom suffer from diabetes. The nursing problems were categorized according to Horta and the interventions according to the NIC. Among the problems found, $76.5 \%$ were of psychobiological needs and $23.5 \%$ were psychosocial. Fifty-three (53) nursing interventions were implemented: $84.9 \%$ were physiological in nature, $11.3 \%$ behavioral and $3.8 \%$ family related. The relationship between the problems identified and the interventions developed were adjusted principally to address the psychobiological needs. Educational interventions were used frequently to attend to the psychosocial needs of the patients.

Descriptors: Nursing; Classification; Nursing care; Mediastinitis.

\section{RESUMEN}

Se trata de estudio cuantitativo, descriptivo y retrospectivo Que objetiva analizar la naturaleza y clasificación de las intervenciones de enfermería para pacientes acometidos por mediastinite en el post-operatorio de cirugía cardiaca. Los resultados logrados a través de prontuarios mostraron una incidencia del $3 \%$ de mediastinite. La población del estudio era en la mayoría del sexo masculino, siendo todos diabéticos. Los problemas de enfermería fueron categorizados según Horta y las intervenciones clasificadas conforme a NIC. Dentro de los problemas estudiados, 76,5\% fueron psicobiológicas y 23,5\% psicosocial, Se implementó 53 intervenciones de enfermería: $84,9 \%$ de naturaleza fisiológica, 11,3\% comportamental y 3,8\% familiar. La relación entre problemas identificados e intervenciones realizadas, fue adecuada principalmente en las necesidades psicobiológicas, siendo frecuente las intervenciones educativas, principalmente en las necesidades psicosociales.

Descriptores: Enfermería; Clasificación; Atención de enfermería; Mediastinitis. 


\section{INTRODUÇÃO}

A evolução da cirurgia cardíaca é marcada pelo contínuo progresso tecnológico e aperfeiçoamento profissional e dentro deste contexto encontra-se a enfermagem, abrindo novas e variadas frentes de atuação com sua assistência fundamentada em ações sistematizadas e inter-relacionadas visando o ser humano, levando ao desenvolvimento de uma tecnologia própria de trabalho com o aprimoramento dos conhecimentos e proposta de novas alternativas de assistência, baseadas em um método científico fundamentado no processo de enfermagem ${ }^{(1)}$.

Apesar dos grandes avanços tecnológicos e do aperfeiçoamento técnico dos profissionais de saúde, as infecções no pós-operatório de cirurgia cardíaca ainda apresentam um grande desafio, uma vez Que ainda constituem causa importante de morbimortalidade nesses pacientes. Dentre essas complicações infecciosas, a mediastinite representa uma das mais graves, determinando um aumento significativo das taxas de morbimortalidade de cirurgias cardíacas, dos custos de internação hospitalar, e, não menos importante, limitação e um grande impacto na vida social dos pacientes Que sobrevivem ${ }^{(2)}$.

A mediastinite é uma infecção profunda da ferida operatória com evidência clínica e/ou microbiológica do comprometimento do espaço retroesternal, associada à osteomielite do esterno com ou em sua instabilidade. É uma entidade com baixa incidência, porém de alta mortalidade ${ }^{(3)}$.

O reconhecimento dos sinais e sintomas da mediastinite aguda torna-se importante para se fazer o diagnóstico precoce, a fim de se iniciar o tratamento o mais rápido possível, evitando-se, assim, a morte do paciente ${ }^{(3)}$.

A responsabilidade de cuidar exige Que as decisões sobre as intervenções propostas sejam fundamentadas na avaliação do estado de saúde do doente. A situação clínica do cliente, a natureza das informações Que se Quer obter e as habilidades cognitivas e perceptivas da enfermagem são fatores Que influenciam a assistência ${ }^{(4)}$.

Quando essa assistência se dá em situações especiais, como em pacientes acometidos por mediastinite no pós-operatório de cirurgia cardíaca, aos três elementos acima citados que influenciam a assistência, associam-se também as maiores probabilidades de ocorrência e identificação de determinadas necessidades de cuidados $^{(4)}$.

Com a expansão do desenvolvimento dos diagnósticos de enfermagem e dos sistemas de classificação, surgiu a necessidade de resgatar e classificar também os tratamentos realizados pela enfermagem, visando comunicar informações de enfermagem para outros elementos da equipe de saúde ou da própria enfermagem.

Embora se tenha a compreensão de Que as classificações dos diagnósticos, resultados e intervenções de enfermagem, bem como as outras classificações existentes, não garantam a incorporação de princípios para a utilização do processo de enfermagem, observandose todas as dimensões Que assegurariam uma prática bem fundamentada, considera-se Que compete aos enfermeiros envolvidos na assistência aos pacientes, acometidos por mediastinite no pósoperatório de cirurgia cardíaca, a busca de novas propostas para o desenvolvimento dos aspectos relativos à sua prática e às iniciativas Que contribuam para uma melhor Qualidade de vida destes pacientes.
De modo particular, o contato com o conteúdo referente à estruturação e às intervenções propostas na Teoria das Necessidades Humanas Básicas e na NIC, despertou a possibilidade de investigação relativa a esse sistema de classificação, uma vez Que se reconhece nestes um trabalho notável de nomeação das ações do enfermeiro.

Assim, espera-se Que os aspectos do conhecimento revelados por este estudo possam ampliar as possibilidades de uma melhor fundamentação do planejamento da assistência de enfermagem e Que sejam utilizados pelos enfermeiros Que atuam no ensino, na pesquisa ou na assistência, trazendo, de alguma forma, benefícios Que alcancem os níveis mais concretos das ações nas situações de interação com os pacientes.

Visto isso, o presente trabalho teve como objetivos analisar a natureza e classificação das intervenções de enfermagem a pacientes acometidos por mediastinite no pós-operatório de cirurgia cardíaca, levantar os problemas de enfermagem apresentados por esses pacientes e classificar essas intervenções de enfermagem.

\section{METODOLOGIA}

Estudo descritivo retrospectivo realizado em um hospital privado de Teresina, PI. A população do estudo foi constituída de seis casos de mediastinite em pacientes submetidos à cirurgia cardíaca nessa instituição, no período de abril de 2003 a abril de 2006.

A coleta de dados deu-se após a autorização da referida instituição e aprovação do Comitê de Ética e Pesquisa da FACID em agosto de 2006, após firmar compromisso com a instituição cenário da pesquisa por meio do Termo de Fiel Depositário.

Os pacientes foram identificados inicialmente na unidade de internação no livro de registros de admissão, alta, transferência e óbitos. Em seguida os prontuários foram identificados Serviço de Arquivo Médico e Estatístico (SAME) da referida instituição e a partir da leitura dos registros e evoluções da equipe de saúde as informações necessárias e adequadas foram registradas no formulário preparado para este estudo. A coleta se deu após firmar compromisso com a instituição por meio do Termo de Fiel Depositário.

Com base nas informações, os problemas identificados foram categorizados conforme proposta de Horta ${ }^{(5)}$ em problemas relacionados às necessidades humanas básicas de ordem psicobiológica, psicossocial e psicoespiritual. As intervenções de enfermagem realizadas foram classificadas de acordo com a Nursing Interventions Classification - $\mathrm{NIC}^{(6)}$.

Os problemas de enfermagem foram denominados com números romanos de I a XVI para facilitar a leitura. As intervenções de enfermagem propostas para os problemas levantados, foram classificadas e codificadas conforme a NIC. Após esse processo de levantamento, classificação e codificação fez-se uma análise Quantitativa desses dados conforme a literatura existente.

\section{RESULTADOS E DISCUSSÃO}

\section{Caracterização da Amostra}

Os resultados deste estudo foram analisados conforme o consolidado e a avaliação das informações colhidas nos prontuários. Para melhor analisar os dados, e atender aos objetivos desse estudo, 
dividiu-se o trabalho em três partes. Inicialmente foi abordada a caracterização da população, em seguida problemas e intervenções de enfermagem, e, por fim, relação entre problemas e intervenções implementadas.

Esta pesquisa foi desenvolvida a partir dos registros em prontuários de seis pacientes, sendo Quatro homens e duas mulheres acometidos por mediastinite no pós-operatório de cirurgia cardíaca. Destes, Quatro pacientes eram do sexo masculino, cinco tinham idade entre 54 e 70 anos e um acima de 70 anos. Vale destacar Que todos eram diabéticos e somente um não era nem hipertenso e nem tabagista.

No período em estudo foram realizadas 198 cirurgias cardíacas, ocorrendo uma incidência de mediastinite de 3,0\%, o Que está de acordo com a literatura, Que diz Que a incidência varia entre 0,2 a $5 \%$ (7).

O período transoperatório é um período crítico para o paciente devido a complexidade da cirurgia cardíaca e procedimentos a ela inerentes, como a Circulação Extra-Corpórea (CEC), e ao tempo intraoperatório prolongado. Com relação às informações referentes à cirurgia, observa-se Que os casos de mediastinite, em um período de três anos, ocorreram em épocas diferentes, o Que reporta a uma fonte não comum de contaminação. Encontrou-se Que a cirurgia de revascularização do miorcárdio foi realizada em cinco pacientes e Que o tempo de CEC em Quatro pacientes foi superior a 90 minutos.

Vários fatores influenciam a incidência de infecção na ferida operatória, entre eles, os procedimentos invasivos e a defesa primária insuficiente provocada pelo trauma cirúrgico e pela CEC. Durante a CEC pode ocorrer desequilíbrio no volume de líeuidos, troca de gases prejudicada, alterações da proteção devido a inibição do sistema de coagulação com a heparinização sistêmica e seQüestro de leucócitos da circulação, inibindo a sua propriedade de fagocitar os germes invasores do organismo ${ }^{(8)}$.

Ao se analisar os resultados constatou-se Que os pacientes passaram em média 11,16 dias na Unidade de Terapia Intensiva e 9,33 horas em ventilação mecânica. Sabe-se Que os hospitais constituem fonte de infecções oportunistas e os microorganismos nosocomiais têm propriedades Que permitem sua sobrevivência no ambiente hospitalar ou no hospedeiro. A Unidade de Terapia Intensiva (UTI), é uma das áreas críticas do ambiente hospitalar, constituindo um reservatório de patógenos com elevado risco de transmissão de infecção a pacientes susceptíveis. Destaca-se Que esses pacientes do estudo permaneceram muito tempo na UTI, além do fato de eles se encontrarem predispostos e possuirem fatores de risco para desenvolver mediastinite ${ }^{(9)}$.

Outro fator Que contribuiu para a longa permanência na UTI e constitui também fator de risco para o desenvolvimento da mediastinite foi a ventilação mecânica prolongada. Na última década, aumentou a importância de se abreviar o tempo de ventilação mecânica em pacientes de pós-operatório de cirurgia cardíaca, baseado nas modificações de técnicas anestésicas e na utilização de novos fármacos opióides Que permitem o despertar mais rápido e, conseQüentemente, extubação e menor tempo na $\mathrm{UTI}^{(10)}$.

Os pacientes acometidos por mediastinite apresentaram sinais e sintomas entre sete e dezoito dias com uma média de 12,16 dias e estes foram: dor na ferida operatória, febre, deiscência, exsudato, hiperemia, instabilidade esternal. Foram submetidos aos seguintes exames diagnósticos: TC de tórax, ecocardiograma e cultura do exsudato sendo identificados os seguintes microorganismos: Stafiloccocus. Aureus em todos os casos além de Pseudomonas e Stafiloccocus Epiderrmidis.

Todos os sintomas apresentados pelos pacientes são comuns a mediastinite e o período de aparecimento dos mesmos está em conformidade com a literatura, considerando que o início dos sintomas se dá entre o $10^{\circ}$ e $15^{\circ}$ dia de pós-operatório ${ }^{(3)}$.

Vale ressaltar a importância do reconhecimento precoce dos sinais e sintomas da mediastinite, como a febre persistente, supuração, dor localizada, entre outros, e a identificação do microorganismo envolvido na infecção, para orientar o tratamento o mais cedo possível, fundamental para o bom prognóstico do paciente.

Devido à mediastinite, os pacientes tiveram uma longa permanência hospitalar, variando entre 21 a 86 dias, com média de 45,67 dias comparando-se à média de internação dos pacientes no pós-operatório de cirurgia cardíaca sem intercorrências nesse mesmo período que foi de sete dias. Esse longo período de internação traz repercussão não só do ponto de vista econômico, mas também psicológico, para o serviço, profissionais de saúde e principalmente para o paciente.

\section{Problemas e Intervenções de Enfermagem}

A partir da análise dos registros de enfermagem nos prontuários, conseguiu-se fazer o levantamento dos problemas de enfermagem apresentados pelos pacientes acometidos por mediastinite em pósoperatório de cirurgia cardíaca no período em estudo.

Os problemas levantados nesta pesQuisa, foram caracterizados de acordo com Horta ${ }^{(5)}$, fazendo-se a ligação com as intervenções classificadas conforme a NIC. Essa ligação, é uma relação entre o diagnóstico e a intervenção de enfermagem para Que ocorram conjuntamente de modo a ser obtido a solução do problema do paciente, fornecendo fundamentação diagnóstica e favorecendo a tomada de decisão(6).

Pela Tabela 2, pode-se constatar Que foram implementadas 53 intervenções de enfermagem para os 16 problemas identificados, sendo estas intervenções de natureza fisiológica $(84,9 \%)$, comportamental $(11,3 \%)$ e família $(3,8 \%)$.

As intervenções de natureza comportamental e familiar apontam para o avanço na direção de um atendimento mais abrangente do paciente, rompendo, assim, um tipo de ação fortemente centrada nos aspectos fisiopatológicos ${ }^{(1)}$.

Ainda vale lembrar Que problema de enfermagem é a situação e/ou condição de assistência apresentada pelo ser humano, pela família ou pela comunidade. Sendo assim, independente do problema do paciente, as intervenções de enfermagem devem abranger e assistir o paciente como um todo ${ }^{(12)}$.

Observando a Tabela 3 , pode-se verificar que $13(76,5 \%)$ dos problemas apresentados pelos pacientes do estudo eram de necessidade psicobiológica, sendo Que foram implementadas, segundo registros nos prontuários, 45 (84,9\%) das ações de enfermagem. Em primeiro lugar, destacam-se as intervenções Que atendem à necessidade de regulação vascular, com as intervenções regulação hemodinâmica e monitorização de lí@uidos. Em seguida, vêm as de integridade física; integridade cutâneo-mucosa; oxigenação, exercício físico e motilidade; eliminação intestinal, 
Tabela 1. Levantamento de problemas de enfermagem apresentados por pacientes acometidos por mediastinite no pósoperatório de cirurgia cardíaca. Teresina - PI, 2006.

\begin{tabular}{clc}
\hline Problemas de Enfermagem & Pacientes \\
\hline I. & Hipertermia & Todos \\
II. & Perfusão tissular ineficaz & Todos \\
III. & Desequilíbrio hidro-eletrolítico & Todos \\
IV. & Desequilíbrio na ventilação-perfusão & Todos \\
V. & Dor aguda & Todos \\
VI. & Mobilidade física prejudicada & Todos \\
VII. & Mobilidade no leito prejudicada & Todos \\
VIII. & Integridade tissular prejudicada & Todos \\
IX. & Regime terapêutico ineficaz & Todos \\
X. & Nutrição deseQuilibrada & Todos \\
XI. & Dificuldade de eliminação intestinal & Todos \\
XII. & Insônia & Todos \\
XIII. & Problema de higiene corporal & Todos \\
XIV. & Desânimo & Todos \\
XV. & Dificuldade respiratória & Apenas IAR \\
XVI. & Saudade da família & Apenas IAR \\
\hline
\end{tabular}

Tabela 2. Natureza e tipos de intervenções identificadas em pacientes acometidos por mediastinite em pós-operatório de cirurgia cardíaca. Teresina - PI, 2006.

\begin{tabular}{lllc}
\hline Natureza & Tipo & $\mathbf{n}$ & \% \\
\hline Fisiológico Básico & & 6 & 13,3 \\
Cuidados Que dão suporte ao funcionamento & Promoção do conforto físico. & 8,9 & 8,9 \\
físico do organismo & Controle da atividade e execução & 4 & 6,7 \\
& Controle da imobilidade & 4 & 6,7 \\
& Suporte nutricional & 3 & 4,3 \\
& Controle das eliminações & 3 & 17,8 \\
Fisiológico Complexo & Facilitação do autocuidado & 2 & 11,1 \\
Cuidados Que dão suporte à regulação & Controle da perfusão tissular & 8,9 \\
homeostática & Controle de pele/lesões & 6,7 \\
& Controle respiratório & 5 & 6,7 \\
Comportamental & Controle de eletrólitos e ácido-básico & 4 & 3 \\
Cuidados Que dão suporte ao funcionamento & Terapia comportamental & 3 & 33,3 \\
psicossocial e facilitam mudanças no estilo de & Assistência no enfrentamento & 2 & 33,3 \\
vida & Promoção do conforto psicológico & 2 & 33,3 \\
Família & Termorregulação comportamental & 2 & 33,3 \\
Cuidados Que dão suporte à unidade familiar & Assistência no enfrentamento & 33,3 \\
& Promoção do conforto psicológico & 2 & 33,3 \\
\hline
\end{tabular}

nutrição, regulação eletrolítica e regulação térmica e, por fim, os de cuidado corporal.

Nessas ações, destacou-se a ênfase dada à orientação sobre a facilitação da auto-responsabilidade e assistência no autocuidado Que pode ser explicada pela crença de Que informado poderá se beneficiar por meio da utilização de medidas Que venham a atenuar os distúrbios na imagem corporal relacionada à lesão, principalmente nesse estudo, no Que se refere à promoção do conforto físico ${ }^{(11)}$.

Outro fato constatado durante o levantamento das intervenções de enfermagem, é que o registro das ações apresenta-se de modo genérico, não constando informações sobre Quem, o Que, onde, Quando, como e com Que freqüência foram realizadas, exceção em algumas ações, como oferecer alívio com analgésicos prescritos, virar conforme indicado pela condição da pele, monitorar as eliminações intestinais, administrar laxante ou enema conforme apropriado. Isso dificultou o levantamento das intervenções. Por esse motivo, utilizou-se a codificação conforme orientação da NIC para se padronizar e evitar, assim, Que se perdessem informações. Além disso, nesse serviço ainda não está implantada a Sistematização da Assistência de Enfermagem (SAE).

Outra característica dessa instituição, também realidade da maioria dos serviços de saúde, é o número insuficiente do profissional enfermeiro, ficando grande parte das intervenções de enfermagem realizadas por auxiliares e técnicos de enfermagem. 
Por isso, muitas das ações realizadas pelos enfermeiros, responsáveis por várias atribuições, algumas de caráter administrativo, são apenas atribuídas verbalmente para a eQuipe de enfermagem executar, não havendo registro adequado das mesmas.

Apesar do sub-registro e da Qualidade de algumas informações, deve-se destacar Que em relação a esse grupo de intervenções, foi onde se encontrou o maior número de ações registradas durante a permanência dos pacientes, bem como uma melhor Qualidade das informações, principalmente nas ações relacionadas às intervenções de oxigenoterapia, cuidados com local da incisão e regulação hemodinâmica. A explicação para esse fato deve-se a preocupação com fatores Que podem causar a QualQuer momento desequilíbrio da homeostasia nesses pacientes Que, por si só, já requerem uma assistência criteriosa, como também, por ser uma instituição particular onde o registro de sinais vitais, administração de medicamentos fora dos horários previamente prescritos, descrição criteriosa da realização dos curativos, entre outros, é uma exigência para se justificar todos os procedimentos.

De acordo com a Tabela 4, Quatro problemas apresentados foram contemplados com oito intervenções Que atendem às necessidades psicossociais, o Que mostra a importância e a necessidade de intervenções de apoio e orientação para o paciente e a família, para controlar ou minorar os efeitos decorrentes da mediastinite na vida do paciente como ser humano único, como membro de uma família e comunidade.
Dentre estas, neste trabalho identificou-se ações voltadas para o controle do comportamento, melhora da imagem corporal, melhora do papel e redução da ansiedade. As ações foram principalmente direcionadas ao aconselhamento e suporte emocional. Justificadas mais uma vez pela necessidade de orientar tanto o paciente como os familiares para se tentar controlar e/ou diminuir os reflexos da mediastinite e sua evolução, deixando-os mais traneüilos, confiantes e receptivos ao tratamento.

Nesses registros, observou-se o desenvolvimento das ações da enfermagem e o seu trabalho em eQuipe com os outros profissionais, como o psicólogo, a inserção desse profissional é importante para o suporte às dimensões psicossociais e psicoespirituais envolvidas no processo de adoecer, tornando a situação mais fácil ${ }^{(11)}$.

Mais uma vez, teve-se grande dificuldade e, porQue não dizer maior, em levantar as ações de necessidade psicossocial, não havendo nenhuma necessidade psicoespiritual registrada. Sabemos Que essas ações são desenvolvidas rotineiramente no cotidiano de enfermagem, até mesmo porQue são esses profissionais Que lidam diretamente com os pacientes e seus familiares, vivenciando situações dessa natureza.

Essa dificuldade encontrada, principalmente no que se refere às necessidades psicossociais, deve-se a vários fatores. Muitas das ações implementadas pela enfermagem acabam não sendo registradas por fazerem parte das orientações habituais ou porQue algumas Questões de âmbito pessoal do paciente ficam restritas ao

Tabela 3. Correspondência entre problemas identificados e intervenções prescritas na área psicobiológica, classificadas de acordo com a NIC, em pacientes com mediastinite em pós-operatório de cirurgia cardíaca. Teresina - PI, 2006.

\begin{tabular}{|c|c|c|}
\hline Necessidades Psicobiológicas & Problemas & Intervenções \\
\hline Exercício e atividade física & Mobilidade física prejudicada & Terapia com exercícios e controle muscular \\
\hline Eliminação intestinal & Dificuldade de eliminação intestinal & Controle da constipação \\
\hline Motilidade & Mobilidade no leito prejudicada & Cuidado com repouso no leito \\
\hline Nutrição & Nutrição desequilibrada & Monitoração nutricional \\
\hline \multirow[t]{2}{*}{ Integridade Física } & Dor aguda & Controle da Dor \\
\hline & Insônia & Controle do Ambiente:Conforto \\
\hline Cuidado Corporal & Problema de higiene corporal & Assistência no auto cuidado: banho/higiene \\
\hline Regulação Eletrolítica & Desequilíbrio hidro-eletrolítico & Controle de $\mathrm{Na}$ e $\mathrm{K}$ \\
\hline Oxigenação & Desequilíbrio na ventilação-perfusão & Oxigenoterapia \\
\hline Integridade Cutâneo-Mucosa & Integridade tissular prejudicada & Cuidados com local da incisão \\
\hline Regulação Térmica & Hipertermia & Tratamento da Febre \\
\hline \multirow[t]{2}{*}{ Regulação Vascular } & Perfusão tissular ineficaz & Regulação Hemodinâmica \\
\hline & Desequilíbrio hidro-eletrolítico & Monitorização de Líuidos \\
\hline
\end{tabular}

Tabela 4. Correspondência entre problemas identificados e intervenções prescritas na área psicossocial, classificadas de acordo com a NIC, em pacientes com mediastinite em pós-operatório de cirurgia cardíaca. Teresina (PI), 2006.

\begin{tabular}{lllc}
\hline Necessidades Psicossociais & Problemas & Intervenções & Código dos Tipos de Ação \\
\hline Aprendizagem (educação à saúde) & Nutrição desequilibrada & Controle do comportamento & 304350.03 \\
& & & 304350.05 \\
Auto-imagem e auto-estima & Dificuldade respiratória & Melhora da imagem corporal & $3 R 5220.01$ \\
& & Melhora do papel & $3 R 5220.03$ \\
Segurança & Saudade da família & Redução da ansiedade & 3 T5820.01 \\
& & & $3 T 5820.03$ \\
Aceitação & Desânimo & Suporte à família & 5X7 I40.04 \\
\hline
\end{tabular}


momento da interação paciente/enfermagem, sem registro posterior dessas orientações, talvez por entenderem Que essas informações não sejam relevantes para o tratamento do paciente.

Sabe-se que o cuidado do paciente é permeado de situações Que envolvem aspectos éticos e a enfermagem deve ter a sensibilidade e o conhecimento necessário para lidar com essas Questões. Além disso, a força do hábito de cuidar de forma assistemática gera lacunas ou mesmo imperfeições em todo o processo assistencial, inclusive na documentação e registro da assistência $^{(11)}$.

No Que tange à relação entre os problemas identificados e as intervenções de enfermagem implementadas, pode-se dizer Que houve certa adequação entre ambos, principalmente aquelas relacionadas às necessidades psicobiológicas.

Vale ressaltar Que as intervenções implementadas são genéricas e, muitas vezes incompletas, e os registros da assistência são escassos, o que dificulta a realização de estudos avaliativos.

Outro aspecto observado foi o não registro dos resultados esperados a partir das ações implementadas o Que gera a falta de parâmetros para a mensuração dos resultados alcançados, comprometendo a avaliação da efetividade das intervenções realizadas. Isso se deve a uma gama de fatores, inclusive a necessária mudança no processo assistencial de enfermagem Que requer intervenções nos âmbito técnico, político e organizacional ${ }^{(11)}$.

\section{CONCLUSÕES}

A realização desse estudo mostrou a importância deste tipo de pesQuisa sobre as intervenções de enfermagem, para se avaliar as ações do enfermeiro no dia-a-dia e propor mudanças necessárias no plano assistencial.

Os principais resultados desse estudo, Que apresentou uma incidência de mediastinite de 3,0\%, está de acordo com a literatura consultada. Houve predomínio de pacientes do sexo masculino e a idade variou entre 54 a 72 anos. Os principais fatores de risco pré-operatório foram diabetes mellitus em todos os casos, hipertensão arterial e tabagismo.

Quanto à cirurgia cinco pacientes foram submetidos à revascularização do miocárdio com tempo de cirurgia acima de cinco horas e duração da circulação extracorpórea acima de 90 minutos. Além dos fatores de risco apresentados pelos pacientes observou-se um longo tempo de permanência na UTI, em média onze dias, e um tempo prolongado em ventilação mecânica.

O Staphyloccocus aureus foi o microrganismo predominante, tendo sido isolado em todos os casos, o Que também está em conformidade com a literatura.

Vale destacar Que esses seis casos de mediastinite ocorreram em períodos diferentes e Que apesar dos vários fatores de risco do pré e pós-operatório, nenhum paciente evoluiu à óbito.

O estudo evidenciou também o predomínio da natureza de intervenções atendendo às necessidades psicobiológicas, havendo também intervenções direcionadas às necessidades psicossociais, principalmente com ações educativas e Que estimulem o autocuidado, demonstrando um avanço nesse aspecto.

A correspondência entre os problemas levantados e as intervenções implementadas foi bem mais evidente no atendimento das necessidades psicobiológicas, devido aos poucos registros, na maioria das vezes genéricos, tanto nesta área como também na psicossocial, não havendo nenhuma ação de enfermagem atendendo necessidades psicoespirituais.

É importante ressaltar Que, os dados levantados nesse estudo mostram a importância da elaboração de um plano assistencial de enfermagem voltado ao atendimento de pacientes submetidos à cirurgia cardíaca, como uma relevante medida para a prevenção e o controle do surgimento de novos casos de mediastinite, contribuindo para práticas de enfermagem seguras e índice de mortalidade zero para esse agravo, como podemos constatar nesse trabalho.

\section{REFERÊNCIAS}

I. Smeltzer SC, Bare BG. Brunner \& Suddarth - Tratado de enfermagem médico-cirúrgica. Rio de Janeiro (RJ): Guanabara Koogan; 1994.

2. Silva RRP. Prevalência de microrganismos isolados em mediastinite pós-cirurgia cardíaca. Rev Mineira Enf 1993; 3(2): 77-80.

3. Auler Júnior JOC. Cirurgia torácica - cirurgia cardiovascular: pós-operatório. Porto Alegre (RS): Artmed; 2004.

4. Cruz DALM, Pimenta CAM. Avaliação do doente com dor crônica em consulta de enfermagem: Proposta de instrumento segundo diagnóstico de enfermagem. Rev Latino-am Enfermagem 1999; 7(3): 49-62.

5. Horta WA. Processo de enfermagem. São Paulo (SP): EPU; 1979.

6. McCloskey IC, Bulechek GM. Classificação das Intervenções de Enfermagem. Porto Alegre (RS): Artes Médicas; 2004.

7. Souza VC, Freire ANM, Tavares-Neto J. Mediastinite pósesternotomia longitudinal. para cirurgia cardíaca: 10 anos de análise. Rev Bras Cir Cardiovasc 2002; 17(3): 266-70.

8. Galdeano LE. Diagnóstico de enfermagem de pacientes no período transoperatório de cirurgia cardíaca. Rev Latino-am Enfermagem 2003; 1 I (2).

9. Pedrosa TMG. Infecção hospitalar. São Paulo (SP): Medsi; 2002.

10. Goldwasser R. Desmame e interrupção da ventilação mecânica. In: David CM. Medicina interna. Rio de Janeiro (RI): Revinter; 2004. p. 441.

11. Gutiérrez MGR. Natureza e classificação das intervenções de enfermagem em ambulatório de Quimioterapia de adultos. Rev Latino-am Enfermagem 2000; 8(3): 33-39.

12. Carraro TE, Westphalen MEA. Metodologia para a assistência de enfermo: teorização, modelos e subsídios para a prática. Goiânia (GO): AB; 2001 . 